\title{
Role of carrier reservoirs on the slow phase recovery of quantum dot semiconductor optical amplifiers
}

Cite as: Appl. Phys. Lett. 94, 041112 (2009); https://doi.org/10.1063/1.3073715

Submitted: 10 November 2008 • Accepted: 31 December 2008 • Published Online: 28 January 2009

J. Kim, C. Meuer, D. Bimberg, et al.

\section{ARTICLES YOU MAY BE INTERESTED IN}

$40 \mathrm{GHz}$ small-signal cross-gain modulation in $1.3 \mu \mathrm{m}$ quantum dot semiconductor optical amplifiers

Applied Physics Letters 93, 051110 (2008); https://doi.org/10.1063/1.2969060

Complete ground state gain recovery after ultrashort double pulses in quantum dot based semiconductor optical amplifier

Applied Physics Letters 90, 033508 (2007); https://doi.org/10.1063/1.2431789

Fast gain and phase recovery of semiconductor optical amplifiers based on submonolayer quantum dots

Applied Physics Letters 107, 201102 (2015); https://doi.org/10.1063/1.4935792

四 QBLOX

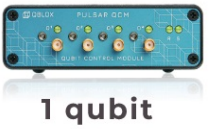

Shorten Setup Time Auto-Calibration More Qubits

Fully-integrated Quantum Control Stacks Ultrastable DC to $18.5 \mathrm{GHz}$ Synchronized $<<1 \mathrm{~ns}$

Ultralow noise

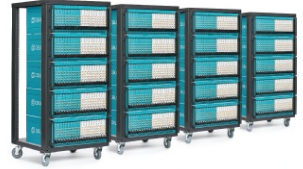

100s qubits

visit our website > 


\title{
Role of carrier reservoirs on the slow phase recovery of quantum dot semiconductor optical amplifiers
}

\author{
J. Kim, ${ }^{1, a)}$ C. Meuer, ${ }^{2}$ D. Bimberg, ${ }^{2}$ and G. Eisenstein ${ }^{3}$ \\ ${ }^{1}$ Department of Information Display, Kyung Hee University, Seoul 130-701, Republic of Korea \\ ${ }^{2}$ Institut fuer Festkoerperphysik, Technische Universitaet Berlin, EW 5-2, Hardenbergstr. 36, \\ 10623 Berlin, Germany \\ ${ }^{3}$ Department of Electrical Engineering, Technion, Haifa 32000, Israel
}

(Received 10 November 2008; accepted 31 December 2008; published online 28 January 2009)

\begin{abstract}
The gain and phase recovery dynamics of quantum-dot (QD) semiconductor optical amplifiers are calculated, including all the optical transitions involved in successive carrier recovery processes. The carrier recovery dynamics of inhomogeneously broadened QDs is simulated by solving 1088 coupled rate equations. The respective contributions of QD states and quantum-well carrier reservoirs to the gain and phase changes are identified both temporally and spectrally. We show that the slow phase recovery component of the QD ground state is induced by the slow carrier dynamics of the carrier reservoir due to a slowly varying line shape function of the refractive index change. (C) 2009 American Institute of Physics. [DOI: 10.1063/1.3073715]
\end{abstract}

Quantum-dot (QD) semiconductor optical amplifiers (SOAs) have drawn much attention due to their picosecond gain recovery time, which can support pattern-effect-free signal amplification up to hundreds of Gbit/s. ${ }^{1-3}$ Phase recovery is also important because it is related with chirping and signal distortion in ultrafast optical pulse amplification. According to the ultrafast pump-probe experiment, the phase recovery, contrary to the gain recovery, is determined by a slow process, which is expected to result from the slow bandfilling of the quantum-well (QW) carrier reservoir. ${ }^{3}$ However, a comprehensive theoretical understanding of the slow phase recovery in QD SOAs is still lacking. In this paper, we theoretically investigate the gain and phase recovery dynamics of QD SOAs by solving about 1000 coupled rate equations and considering all the optical transitions involved in the carrier recovery processes. The ultrafast gain and phase recovery responses, induced by four consecutive optical pulses, are calculated in both spectral and time domain from low to high current densities. We identify how the respective carrier dynamics of the QD states and the QW reservoir contributes to the fast and slow phase recovery components of QD SOAs.

The gain and phase recovery dynamics is determined by the carrier relaxation time constants and the spectral line shape functions of all the optical transitions involved in the successive carrier recovery processes. Figure 1(a) shows the schematic diagram of the carrier recovery process together with the respective time constants. After a carrier at the QD ground state (GS) is instantaneously depleted due to signal amplification, the empty GS is refilled by the carrier relaxation from the QD excited state (ES) within less than 1 ps. $^{1-3}$ The empty ES is refilled by the carrier capture from the QW layer on a time scale of around $3 \mathrm{ps} .{ }^{2}$ Finally, the QW carrier reservoir is recovered to reach equilibrium of carrier distribution in the time range of 0.1-1 ns. ${ }^{4}$ Figure 1(b) shows two optical transition mechanisms of the interband transition (dotted line) and intraband free carrier absorption (solid line). In the interband transition, a pair of electron and hole is generated or recombines, which induces the gain and refrac-

${ }^{a)}$ Electronic mail: junghokim@khu.ac.kr. tive index change related with the density of states, the homogeneous line shape function, and the carrier occupation probability. In the intraband free carrier absorption, an electron or a hole alone absorbs a photon and transits to a higherlying continuum state. In general, the refractive index change due to the free carrier absorption from the QW layer or the QD states to continuum states can be modeled by the classical Drude model of free carrier plasma. ${ }^{5,6}$

Figure 2 shows the schematic diagram of inhomogeneously broadened QDs, where the QD ensemble is partitioned into $M=181$ spectral groups with an energy interval of $\Delta E=1 \mathrm{meV}$ (Ref. 7) to cover the wavelength range between 1100 and $1400 \mathrm{~nm}$. The maximal distributions of the GS and the ES ensembles are located at 1290 and $1190 \mathrm{~nm}$, respectively. As shown in the inset, the carrier dynamics of electron and hole are considered separately. Each QD has two spin-degenerate GS and a fourfold degenerate ES, and a many-fold upper state (US). The InGaAs QW layer works as a common carrier reservoir for all sizes of QDs. Total 1088 $(181 \times 3+1$ for electron, $181 \times 3+1$ for hole $)$ coupled rate equations are numerically solved by the Runge-Kutta method. The arrows in the inset represent the carrier relaxation and escape processes between QD states. The capture and relaxation times of electron are assumed to be 3 and 1 ps, respectively. Both capture and relaxation times of hole are assumed to be 0.13 ps to reflect the faster hole relaxation in QDs. We use the Lorentzian line shape function with a full width at half maximum of $10 \mathrm{meV}$ to include the gain and refractive index change due to the interband transitions. The modal refractive index change induced by the free carrier absorption is obtained by ${ }^{5,6} \Delta n_{\text {free }}=-\Gamma e^{2} N /\left(2 \varepsilon_{0} n_{b} m^{*} \omega^{2}\right)$, where $e$ is the elementary charge, $\varepsilon_{0}$ is the permittivity in free space, $n_{b}$ is the background refractive index, $\omega$ is the optical frequency, and $N$ is the carrier density at each QD and QW state. The term $\Gamma$ is the optical confinement factor and $m^{*}$ is the effective mass of electron or hole, whose values are different for InAs QDs and InGaAs QWs.

Figure 3 shows the modal gain and the differential modal gain spectra at the injection current density of 0.4 and $1.0 \mathrm{kA} / \mathrm{cm}^{2}$, which correspond to low and high current densities. The difference of the modal gains between the injec- 


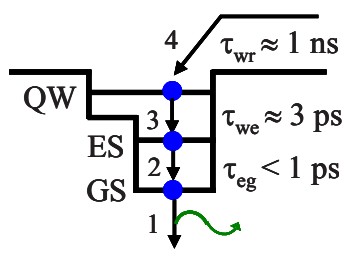

(a)

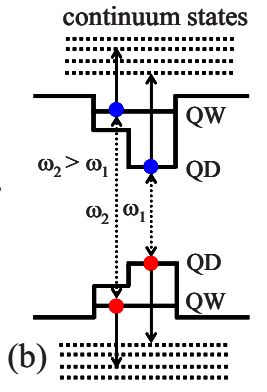

FIG. 1. (Color online) (a) Schematic diagram of the successive carrier recovery process together with the respective time constants. (b) Two optical transition mechanisms of the interband transitions (dotted line) and free carrier absorptions (solid line)

tion current densities of $0.3(0.9)$ and $0.4(1.0) \mathrm{kA} / \mathrm{cm}^{2}$ is used for the differential modal gain. When the injection current density is $0.4 \mathrm{kA} / \mathrm{cm}^{2}$, the carrier reservoir such as the ES is not fully occupied such that most of differential modal gain change takes place at the ES as shown in Fig. 3(a). When the injection current density increases up to $1.0 \mathrm{kA} / \mathrm{cm}^{2}$, the optical gain of the GS and the ES becomes nearly saturated and the differential modal gain change mostly occurs at the US and the QW, which is not shown in Fig. 3(b). Due to the fast decaying tail of the gain lineshape function, the GS gain changes induced by the US and the QW will be negligible. On the contrary, the differential refractive index changes due to the US and the QW significantly contribute to the refractive index change at the GS as shown in Fig. 4. This results from the slowly varying tail of

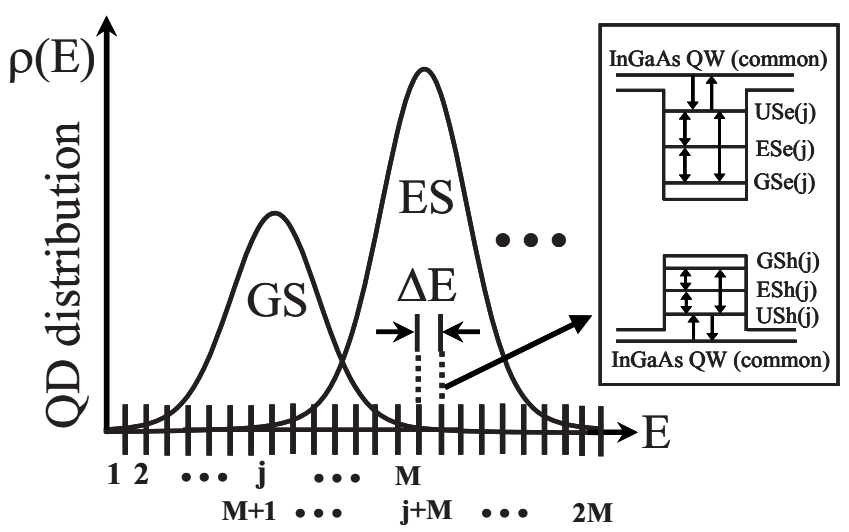

FIG. 2. Schematic diagram of inhomogeneously broadened QDs. Inset: the energy diagram of one QD.

the line shape function for refractive index changes.

To simulate the ultrafast gain and phase recovery, we assume that four consecutive pump pulses with the time interval of 5 ps is injected into a QD SOA. Each pump pulse has a rectangular shape with the width of $150 \mathrm{fs}$ and the energy of $0.65 \mathrm{pJ}$. The wavelength of the pump is around $1290 \mathrm{~nm}$, which is the peak wavelength of the GS ensemble. The coupled rate equations are solved to calculate temporal variations in the carrier densities at each QD group, from which the optical gain and refractive index change are obtained. Finally, the temporal changes in the gain $(\Delta G)$ and the phase $(\Delta \phi)$ are calculated by $\Delta G=\exp \left[P_{\mathrm{prb}}(t\right.$ $\left.\geq 0) / P_{\mathrm{prb}}(t<0)\right]$ and $\Delta \phi=-(2 \pi / \lambda) \times L[n(t \geq)-n(t<0)]$, re-
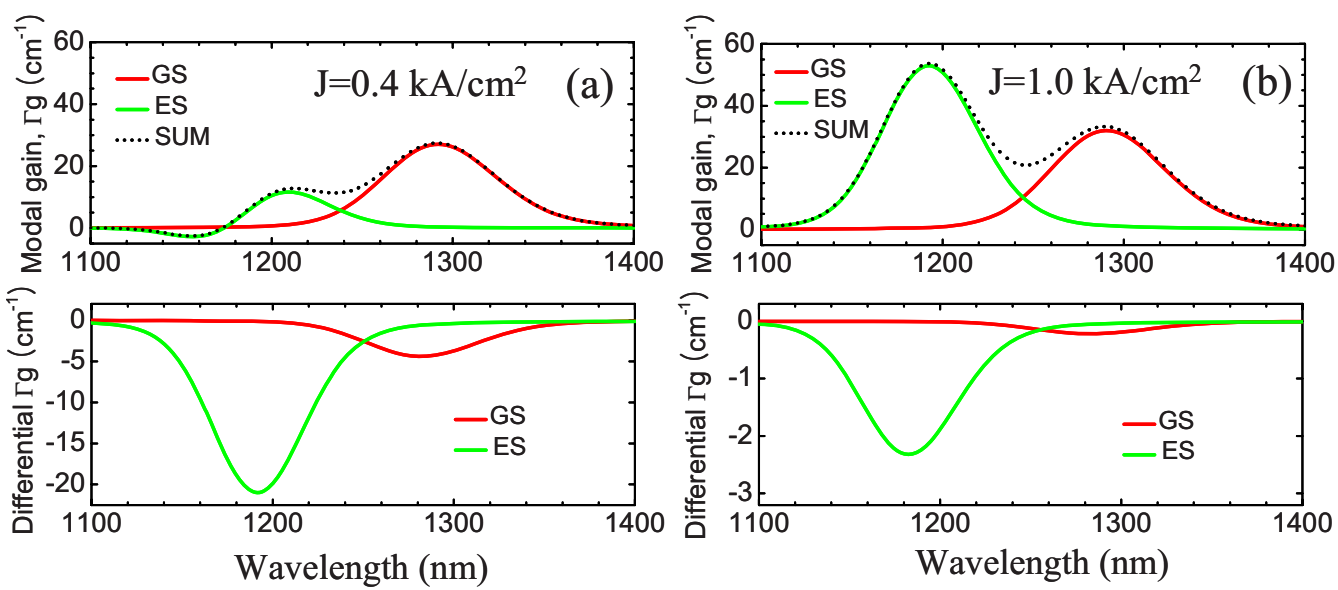

FIG. 3. (Color online) Modal and differential modal gain spectra at the injection current density of (a) 0.4 and (b) $1.0 \mathrm{kA} / \mathrm{cm}^{2}$.
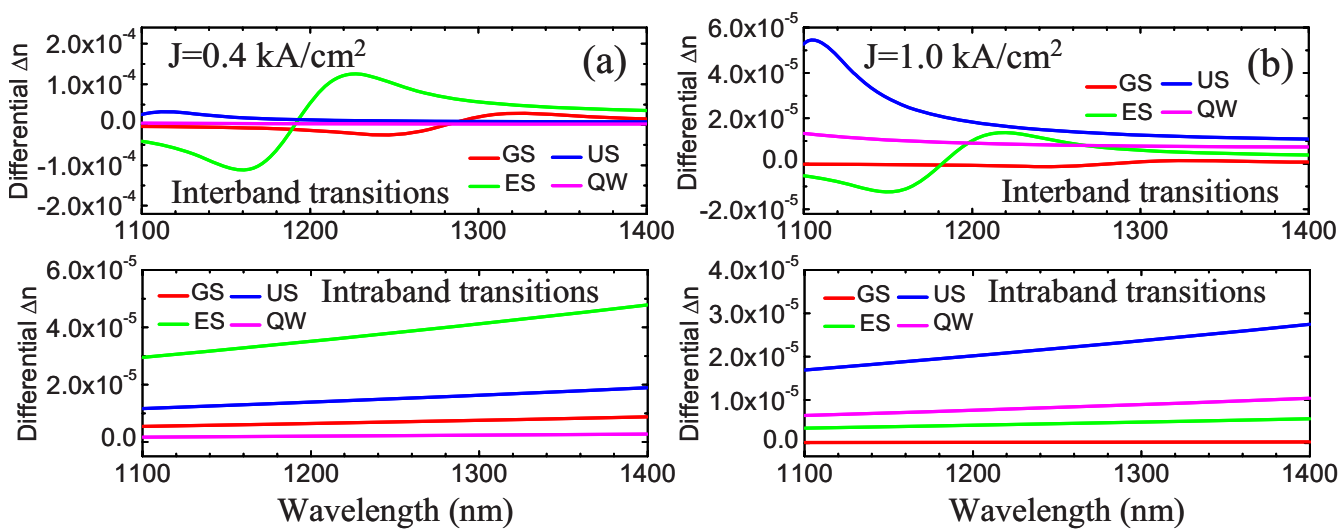

FIG. 4. (Color online) Differential refractive index change spectra due to (upper) interband and (lower) intraband free carrier transitions at the injection current density of (a) 0.4 and (b) $1.0 \mathrm{kA} / \mathrm{cm}^{2}$. 

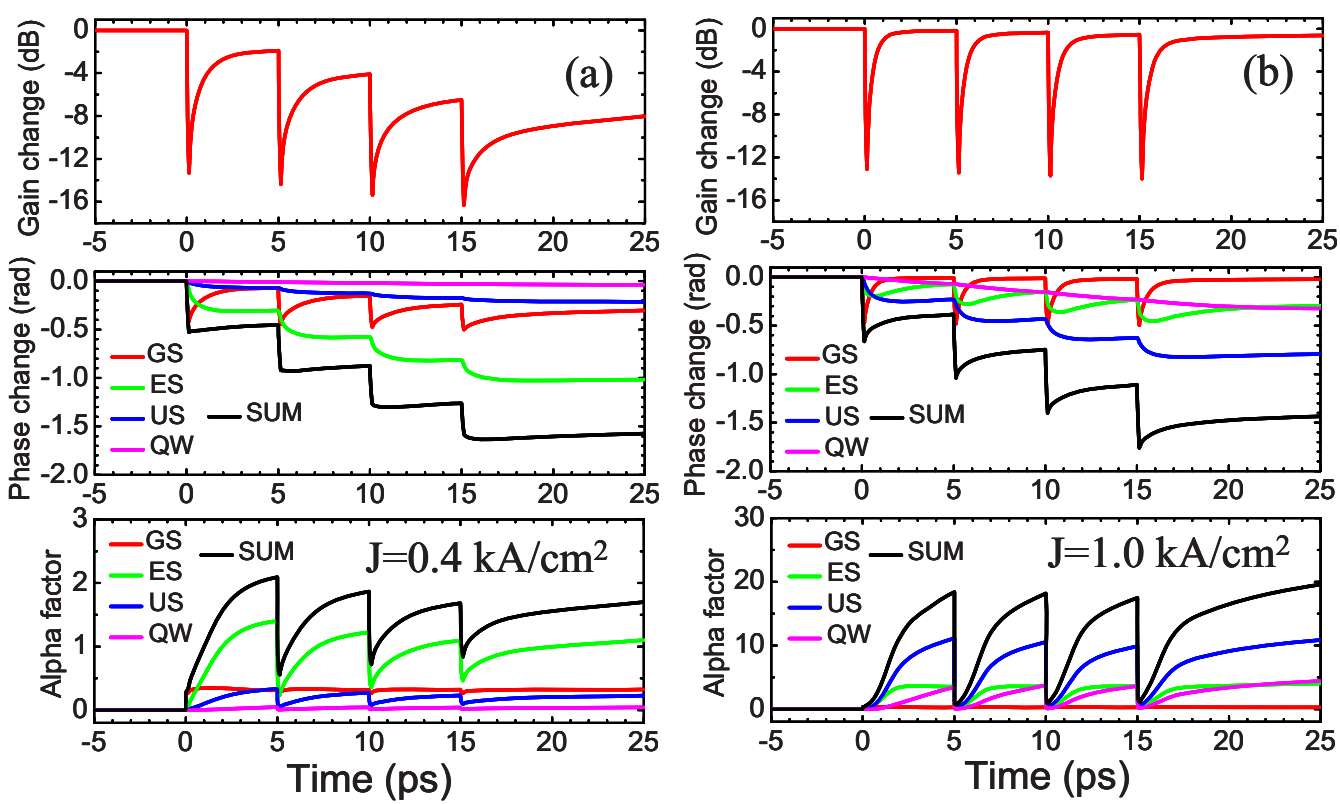

FIG. 5. (Color online) Calculated temporal variations in the gain change, the phase change, and the $\alpha$ factor at the probe wavelength of $1292 \mathrm{~nm}$ at the injection current density of (a) 0.4 and (b) $1.0 \mathrm{kA} / \mathrm{cm}^{2}$. The respective contributions of the GS, the ES, the US, and the QW are marked in different colors.

spectively. The alpha factor $(\alpha)$ is obtained by the relation $\alpha=2 \Delta \phi / \Delta G$. Here, $P_{\text {prb }}$ is the output probe power and $n$ is the modal refractive index change. The term $L$ is the length of the SOA, which is $2 \mathrm{~mm}$ in this calculation. The other parameters can be found elsewhere. ${ }^{7}$

Figure 5 shows the calculated temporal variations in the gain change, the phase change, and the $\alpha$ factor at the probe wavelength of $1292 \mathrm{~nm}$ when the injection current density is 0.4 and $1.0 \mathrm{kA} / \mathrm{cm}^{2}$. The respective contributions of the GS, the ES, the US, and the QW are marked in different colors. As the injection current density increases from 0.4 to $1.0 \mathrm{kA} / \mathrm{cm}^{2}$, the GS gain recovery is significantly accelerated due to the enhancement of ultrafast carrier relaxation from the carrier reservoirs. The fast phase-recovery component caused by ultrafast carrier relaxation from the carrier reservoirs, cannot be observed in bulk SOAs, ${ }^{8,9}$ is also accelerated at high current injection. However, the slow phaserecovery caused by the slow band-filling of the US and the QW, similar to bulk SOAs, ${ }^{8,9}$ is not accelerated at high injection current due to the slowly varying line shape function of the refractive index change, as shown in Fig. 4. Thus, the calculated phase change looks like a stair shape, where a certain amount of the phase change is accumulated whenever the next pump pulse is injected. If the same amount of carriers is depleted at the same wavelength from QD and bulk SOAs with the same optical gain, the magnitude of the slow phase change in QD SOAs will be smaller than that in bulk SOAs because the slow phase change in the GS in QD SOAs is induced by a slowly varying tail of the line shape function of the refractive index change. The alpha factor at the low current density is less than two. However, the alpha factor at the high current density has a very large value of more than ten because the main recovery mechanism of the gain and phase changes is different at the high current injection. These calculated results in Fig. 5 agree to the experimental measurements. ${ }^{1,3}$

In conclusion, we present the numerical results of the gain and phase recovery dynamics of QD SOAs by solving
1088 coupled rate equations. As the injection current increases, the GS gain recovery is significantly improved by the enhancement of fast carrier relaxation from the carrier reservoirs. On the other hand, the GS phase recovery still has a slow component at high current density due to a slowly varying tail of the refractive-index line shape function from the higher-lying QW carrier reservoirs. However, this slow phase change in QD SOAs is relatively smaller than that of bulk SOAs, which can be one of the advantages of QD SOAs. We expect that this slow phase recovery component can be further reduced by increasing the homogeneity or the barrier potential of QDs.

This work was supported by the TRIUMPH project (Project No. IST-027638 STP) and the Sonderforschungsbereich SFB 787 of the German Research Council. The work of J.K. and G.E. was supported by the Alexander von Humboldt foundation. The work of J.K. was also supported by the European Union under the Marie Curie Fellowship (Grant No. MIF1-CT-2006-040250) at the Technical University of Berlin, 10623 Berlin, Germany.

\footnotetext{
${ }^{1}$ S. Dommers, V. V. Temnov, U. Woggon, J. Gomis, J. Martinez-Pastor, M. Laemmlin, and D. Bimberg, Appl. Phys. Lett. 90, 033508 (2007).

${ }^{2}$ I. O'Driscoll, T. Piwonski, C.-F. Schleussner, J. Houlihan, G. Huyet, and R. J. Manning, Appl. Phys. Lett. 91, 071111 (2007).

${ }^{3}$ T. Vallaitis, C. Koos, R. Bonk, W. Freude, M. Laemmlin, C. Meuer, D. Bimberg, and J. Leuthold, Opt. Express 16, 170 (2008).

${ }^{4}$ J. Mørk, M. L. Nielsen, and T. W. Berg, Opt. Photonics News 14, 42 (2003).

${ }^{5}$ C. H. Henry, R. A. Logan, and K. A. Bertness, J. Appl. Phys. 52, 4457 (1981).

${ }^{6}$ A. V. Uskov, E. P. O’Reilly, D. McPeake, N. N. Ledentsov, D. Bimberg, and G. Huyet, Appl. Phys. Lett. 84, 272 (2004).

${ }^{7}$ C. Meuer, J. Kim, M. Laemmlin, S. Liebich, A. Capua, G. Eisenstein, A. R. Kovsh, S. S. Mikhrin, I. L. Krestnikov, and D. Bimberg, Opt. Express 16, 8269 (2008).

${ }^{8}$ R. Giller, R. J. Manning, and D. Cotter, IEEE Photon. Technol. Lett. 18, 1061 (2006).

${ }^{9}$ J. Wang, A. Maitra, C. G. Poulton, W. Freude, and J. Leuthold, J. Lightwave Technol. 25, 891 (2007).
} 